\title{
Documentation of Engraftment and Chimerism After HSCT
}

\author{
Peter Bader
}

\subsection{Introduction}

It is of central interest to document that the newly developing hematopoiesis post-transplant is of donor or recipient origin. The investigations of the genotype origin of post-transplant hematopoiesis are called chimerism analysis. The term "chimerism" was first introduced into medicine in 1951. Andresen wrote that an organism with cells from two or more distinct zygote lineages is a "chimera." Since 1956 this term was used in field of transplantation (Ford et al. 1956). Chimera refers itself to the Greek mythology where Homer described a fire-spitting monster with the head of a lion, a tail of a serpent, and the body of a goat terrorizing Lycia, a region in Minor Asia.

For a long time, it was believed that complete donor hematopoiesis is necessary to maintain engraftment after allo-HSCT. A few decades ago, it became apparent that donor and recipient hematopoiesis may coexist. This state of coexistence of hematopoietic cells is called mixed chimerism (MC). If all cells are of donor origin, the patient is referred to as "complete chimera," and he shows a "complete chimerism."

P. Bader $(\bowtie)$

Division for Stem Cell Transplantation and Immunology, University Hospital for Children and Adolescents, Goethe University Frankfurt am Main, Frankfurt, Germany e-mail: peter.bader@kgu.de
It is important to note that the state of hematopoietic chimerism may underlay a certain dynamic. Patients with a complete chimerism may develop a "mixed chimerism" at a later time point or vice versa. In the later patients, the amount of autologous cells may "increase" or "decrease." The patients then develop an "increasing mixed chimerism" or a decreasing mixed chimerism. To avoid misunderstandings as to whether donor or recipient hematopoiesis changes, it is recommended to report "increasing mixed donor chimerism" or "increasing mixed recipient chimerism."

Nowadays, it has become possible to analyze hematopoietic chimerism also in single cell subpopulations. If a patient's hematopoiesis is mixed only in different cell lines, these patients are referred to have a "split chimerism." Finally the applied method for chimerism analysis has also an impact on the degree of chimerism. A patient could be complete chimera with a method detecting about $1 \%$ autologous cells, whereas recipient cells could have been detected with a more sensitive technique (Bader et al. 2005).

\subsection{Methods for Chimerism Analysis}

Different methods have been developed for the assessment of hematopoietic chimerism. All these methods followed the same principle using 
differences in polymorphic genetic markers and their products. Historically restriction fragment length polymorphism (RFLP), cytogenetics, red cell phenotyping, and fluorescence in situ hybridization techniques were used for the assessment of hematopoietic chimerism. All of these techniques have been very time-consuming and did not always offer the possibility to be used in every patient-donor constellation.

Widespread and timely clinical applicability has become possible after polymerase-chainreaction (PCR) techniques were developed. During the 1990s, these analyses were mainly performed by amplification of variable number of tandem repeats (VNTR). Later in the decade short tandem repeats (STR) were used. Fluorescent labeling of the primers and resolution of PCR products with capillary electrophoresis allowed immediate and accurate quantification of the degree of chimerism. Semiautomated PCR analysis using the appropriate hardware allowed moreover high sample throughput. This made it possible to study chimerism in all patients and in short time intervals already early after transplantation. Accurate monitoring of engraftment as well as surveillance of impending graft rejection in patients transplanted for nonmalignant disease has become possible (McCann and Lawler 1993; Alizadeh et al. 2002; Thiede et al. 2001).

Recently, real-time PCR (rPCR) approaches using single nucleotide polymorphism (SNP) have also become available for the detection of chimerism. SNPs are biallelic variants that differ from each other only at a single nucleotide and occur on average every $1.3 \mathrm{~kb}$ in the human genome. This rPCR has an even higher sensitivity compared to STR-PCR assays, but their quantitative accuracy with a variation coefficient of only $30-50 \%$ is lower compared to $1-5 \%$ of the STR systems.

The latest developments for the detection of chimerism are the analysis using digital PCR (dPCR) procedures. This technology allows accurate and absolute quantification of DNA. This dPCR system is based on deletion/insertion polymorphism (DIP/INDEL) analysis. Clinical studies using this technique, however, are not yet performed (Jacque et al. 2015; Clark et al. 2015).
Based on these issues, the STR-PCR with fluorescent-labeled primers and resolution of the fragments with capillary electrophoresis is currently still considered to be the gold standard for the assessment of post-transplant chimerism. It is important to stress that whatever method is employed to study chimerism, it is important that the procedure is standardized and the chimerism laboratory is accredited and is participating in quality control rounds (Lion et al. 2012).

\subsection{Chimerism Investigation in the Clinical Setting}

\subsubsection{Chimerism in Nonmalignant Diseases}

Allo-HSCT is the only curative treatment option for many patients with inherited or acquired nonmalignant diseases such as immunodeficiency, storage diseases, osteopetrosis, thalassemia, sickle cell disease, severe aplastic anemia, bone marrow failure syndromes, and many others.

The aim of the transplant procedure in these diseases is to achieve stable and durable engraftment to (1) improve the hematopoietic function, to (2) correct the immune competence, and/or to (3) increase or normalize the respective enzyme shortage. As a consequence, it is not always necessary to replace the recipient hematopoiesis completely. For many diseases, it is sufficient to implement a state of mixed hematopoietic chimerism to improve the patients' well-being. To minimize toxic side effects intensity of conditioning regimens in these diseases is often reduced and therefore less myeloablative. MC is more likely, and graft rejection or non-engraftment remained the major causes of treatment failures in these patients (Bader et al. 2005; Thiede et al. 2001).

It could be shown that rapid donor cell presence and maintenance of early complete donor chimerism in NK and T cells may play an important role in achieving sustained engraftment especially in patients who were treated with reduced intensity conditioning regimens. Analysis of chimerism in disease characterizing 
cell subpopulations in patients with nonmalignant disease, e.g., in patients with severe combined immune deficiencies (SCID) or in patients with storages disease, enables the documentation of success of the transplant procedures (Preuner et al. 2016).

\subsubsection{Intervention to Influence the Evolution of Chimerism: Transfusion of DLI}

In patients with nonmalignant diseases, MC occurs frequently. The question whether individual patients with MC are at risk to reject their graft depends on the diagnosis and on the conditioning regimens. Studies have clearly shown that MC can be influenced by DLI. MC can be stabilized or even converted to complete donor chimerism by DLI. However, in treating patients with MC and DLI, physicians have to bear in mind the potential risk to induce GVHD which should be avoided in patients with nonmalignant disease with all efforts.

\section{Hemoglobinopathies}

In thalassemia patients, large studies have been published already from the Pesaro group of Guido Lucarelli, evaluating the influence of MC and disease recurrence. In general it was found that patients whose recipient $\mathrm{MC}$ increased to $>30 \%$ autologous cells were by far more likely to ultimately reject and be transfusion dependent. However, there are patients with persisting high level MC who remained transfusion independent. Retrospective studies have been performed evaluating the possibility of influencing $\mathrm{MC}$ by DLI. It could be shown that a state of MC may be sufficient to remain transfusion independent. It was also shown that DLI is capable to convert MC to CC. However, no general recommendation could be given at the time being (Fitzhugh et al. 2014; Karasu et al. 2012; Abraham et al. 2017).

In sickle cell disease (SCD), the impact of MC has been studied intensively as more and more patients with SCD were transplanted from matched but also from mismatched donors. In the late 1990s, first studies concluded that $10 \%$ of donor cell engraftment and persistence were needed for effective treatment of SCD in patients who were treated with a homozygous healthy donor; however, if the patient was grafted with the stem cells of a heterozygous HbAS donor, $30-40 \%$ donor cells are required. The presence of MC in patients transplanted for sickle cell disease does not warrant DLI per se. In patients with less than $30 \%$ of donor chimerism, DLI might be considered. In a most recent study, Fitzhugh and colleagues developed a mathematical model by which they could show that a donor chimerism in the myeloid compartment of $20 \%$ is necessary to reverse the sickle cell phenotype and to prevent patients from disease recurrence (Fitzhugh et al. 2017).

\subsubsection{Chimerism in Malignant Diseases}

Chimerism detected by molecular methods allows the assessment of persisting or reappearing recipient cells after allo-HSCT. These cells might be a reflection of either survival of malignant cells or of survival or recurrence of recipient hematopoietic cells or a combination of both. It could be shown by prospective studies already in the early 1990s that a MC frequently occurs in the mononuclear cell fraction, weakens thereby the GvL effect, and facilitates recurrence of the underlying leukemia.

Chimerism analysis does provide information about the alloreactivity and/tolerance induction of the graft and thereby serves more likely a "prognostic factor" than as an indirect marker for MRD. It has become evident that the development of post-transplant chimerism is a dynamic process. Hence, if chimerism analyses are performed in the intention to detect impending relapse, investigations need to be performed in short time intervals (Bader et al. 2004b; Thiede et al. 2001; Kröger et al. 2010a, b).

Initially, many pediatric studies using serial analysis of chimerism could clearly demonstrate that patients who develop a MC Post transplant have an increased risk for future relapse of their leukemia. This could later also be confirmed by studies in adult patients. 
Moreover these and subsequent studies undoubtedly showed that by immunotherapeutic interventions, e.g., withdrawal of IS or transfusion of DLI, MC could be converted to complete chimerism, GvL effect restored, and many patients prevented from developing overt hematological relapse (Platzbecker et al. 2012; Bader et al. 2004a).

Based on its limited sensitivity to detect minor cell population of about $1 \%$, chimerism analysis in the whole blood is not suitable to serve as a MRD marker. For the assessment of MRD, other techniques should be used, if possible. In patients and diseases lacking a disease-specific marker, for example, regularly in patients with MDS and often in patients with AML, chimerism analysis could be performed in cell subpopulations. Thiede et al. could clearly demonstrate that by the characterization of chimerism in the CD34-positive cell fraction, leukemia relapse could be anticipated in advance in many patients with AML and MDS. In ALL patients, several studies have been performed investigating the impact of MC after enrichment of entity specific subpopulation, e.g., CD 10, CD19, and CD 34 for precursor B ALL and CD3, CD4, CD5, and CD8 for T-lineages. Remarkable correlation between MRD and chimerism in different subsets could be proven (Platzbecker et al. 2012; Bornhäuser et al. 2009; Rettinger et al. 2011).

Serial and quantitative analysis of chimerism allows the identification of patients at highest risk for relapse. Not all patients can be identified, and time interval between the onset of MS and relapse is often short. It is essential to perform the analysis frequently and ideally: chimerism should be combined with MRD analysis to optimize the predictive value. These investigations can form the basis for individual preemptive immunotherapy strategies to prevent recurrence of the underlying disease.

\section{Key Points}

- Documentation of engraftment is the important step on the way to successful HSCT

- Post-transplant patients are carrying two different genetic profiles and are called chimera

- Analysis of hematopoietic chimerism offers the possibility to realize impending graft rejection and may also serve as an indicator for the recurrence of the underlying disease

- Since several years, these investigations have become the basis for intervention strategies to:

- Avoid graft rejection

- Maintain engraftment

- To treat imminent relapse by preemptive immunotherapy

\section{References}

Abraham A, Hsieh M, Eapen M, et al. Relationship between mixed donor-recipient chimerism and disease recurrence after hematopoietic cell transplantation for sickle cell disease. Biol Blood Marrow Transplant. 2017;23:2178-83.

Alizadeh M, Bernard M, Danic B, et al. Quantitative assessment of hematopoietic chimerism after bone marrow transplantation by real-time quantitative polymerase chain reaction. Blood. 2002;99:4618-25.

Bader P, Kreyenberg H, Hoelle W, et al. Increasing mixed chimerism is an important prognostic factor for unfavorable outcome in children with acute lymphoblastic leukemia after allogeneic stem-cell transplantation: possible role for immuno-therapy. J Clin Oncol. 2004a;22:1696-705.

Bader P, Kreyenberg H, Hoelle W, et al. Increasing mixed chimerism defines a high-risk group of childhood acute myelogenous leukemia patients after allogeneic stem cell transplantation where pre-emptive immunotherapy may be effective. Bone Marrow Transplant. 2004b;33:815-21. 
Bader P, Niethammer D, Willasch A, et al. How and when should we monitor chimerism after allogeneic stem cell transplantation? Bone Marrow Transplant. 2005;35:107-19.

Bornhäuser M, Oelschlaegel U, Platzbecker U, et al. Monitoring of donor chimerism in sorted CD34+ peripheral blood cells allows the sensitive detection of imminent relapse after allogeneic stem cell transplantation. Haematologica. 2009;94:1613-7.

Clark JR, Scott SD, Jack AL, et al. Monitoring of chimerism following allogeneic haematopoietic stem cell transplantation (HSCT). Br J Haematol. 2015;168:26-37.

Fitzhugh CD, Abraham A, Hsieh MM. Alternative donor/ unrelated donor transplants for the $\beta$-thalassemia and sickle cell disease. Adv Exp Med Biol. 2017;1013:123-53.

Fitzhugh CD, Abraham AA, Tisdale JF, et al. Hematopoietic stem cell transplantation for patients with sickle cell disease. Hematol Oncol Clin North Am. 2014;28:1171-85.

Ford CE, Hamerton JL, Barnes DW, et al. Cytological identification of radiation-chimaeras. Nature. 1956;177:452-4.

Jacque N, Nguyen S, Golmard J-L, et al. Chimerism analysis in peripheral blood using indel quantitative realtime PCR is a useful tool to predict post-transplant relapse in acute leukemia. Bone Marrow Transplant. 2015;50:259-65.

Karasu GT, Yesilipek MA, Karauzum SB, et al. The value of donor lymphocyte infusions in thalassemia patients at imminent risk of graft rejection following stem cell transplantation. Pediatr Blood Cancer. 2012;58:453-8.

Kröger N, Bacher U, Bader P, et al. NCI first international workshop on the biology, prevention, and treat- ment of relapse after allogeneic hematopoietic stem cell transplantation. Biol Blood Marrow Transplant. 2010a;16:1325-46.

Kröger N, Bacher U, Bader P, et al. NCI first international workshop on the biology, prevention, and treatment of relapse after allogeneic hematopoietic stem cell transplantation. Biol Blood Marrow Transplant. 2010b;16:1187-211.

Lion T, Watzinger F, Preuner S, et al. The EuroChimerism concept for a standardized approach to chimerism analysis after allogeneic stem cell transplantation. Leukemia. 2012;26:1821-8.

McCann SR, Lawler M. Mixed chimaerism; detection and significance following BMT. Bone Marrow Transplant. 1993;11:91-4.

Platzbecker U, Wermke M, Radke J, et al. Azacitidine for treatment of imminent relapse in MDS or AML patients after allogeneic HSCT. Leukemia. 2012;26:381-9.

Preuner S, Peters C, Pötschger U, et al. Risk assessment of relapse by lineage-specific monitoring of chimerism in children undergoing allogeneic stem cell transplantation for acute lymphoblastic leukemia. Haematologica. 2016;101:741-6.

Rettinger E, Willasch AM, Kreyenberg H, et al. Preemptive immunotherapy in childhood acute myeloid leukemia for patients showing evidence of mixed chimerism after allogeneic stem cell transplantation. Blood. 2011;118:5681-8.

Thiede C, Bornhäuser M, Oelschlägel U, et al. Sequential monitoring of chimerism and detection of minimal residual disease after allogeneic blood stem cell transplantation (BSCT) using multiplex PCR amplification of short tandem repeat-markers. Leukemia. 2001;15:293-302.

Open Access This chapter is licensed under the terms of the Creative Commons Attribution 4.0 International License (http://creativecommons.org/licenses/by/4.0/), which permits use, sharing, adaptation, distribution and reproduction in any medium or format, as long as you give appropriate credit to the original author(s) and the source, provide a link to the Creative Commons license and indicate if changes were made.

The images or other third party material in this chapter are included in the chapter's Creative Commons license, unless indicated otherwise in a credit line to the material. If material is not included in the chapter's Creative Commons license and your intended use is not permitted by statutory regulation or exceeds the permitted use, you will need to obtain permission directly from the copyright holder.

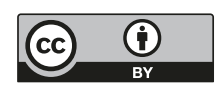

